\title{
AN APPROACH TO ASSESS THE IMPACT OF EXTREME EXTERNAL INFLUENCES ON THE FUNCTIONALITY OF SPECIALIZED DEVICES
}

\author{
NiKolAY L. GEORGIEV* \\ Institute of Metal Science, Equipment and Technologies with Hydro- and \\ Aerodynamics Centre "Acad. A. Balevski", Bulgarian Academy of Sciences, \\ 67, Shipchenski prohod St., 1574 Sofia, Bulgaria
}

[Received: 08 April 2019. Accepted 08 July 2019]

doi: 10.7546/JTAM.49.19.03.06

\begin{abstract}
The presented study proposes an approach to accelerate the tests intended to assess the impact of extreme external influences on the functionality of specialized devices. For this purpose, models are proposed for describing the probability of failure-free operation of specialized devices in areas close to the limit values of the external effects parameters for which they are designed. Examples are given for applying the approach to assess the impact of extreme external temperature and mechanical impacts.
\end{abstract}

KEY WORDS: specialized sensor, external influences, factor, model.

\section{INTRODUCTION}

The concept of "specialized devices" is becoming increasingly popular however it is not clearly defined in the existing literature. In most cases, this expression is used for devices that meet at least one of the two conditions: they are intended for use in an environment where external influences (temperature, radiation, vibrations, etc.) differ significantly from the standard (so-called "extreme external influences"), or have functional characteristics exceeding those of the most devices of the type (e.g. ultra-precision, tiny- or over-sized, exceptionally light or heavy, etc.) [1-4].

For the purpose of this study, under "specialized devices" we will understand those that meet the first of these conditions, i.e. devices intended for use in an environment where external influences are significantly different from the standard ones. The environment in which this type of specialized devices operates affects their working capacity through the impact of a number of factors that are described by some authors as part of the so-called objective destabilizing factors. The main ones are the temperature and mechanical impacts (mainly vibrations, accelerations and shocks).

The most commonly used approach in assessing the influence of external influence parameters on the performance of specialized sensors is to test the latter at normal and extreme values of these influences [1-7]. For example, if the specialized

\footnotetext{
${ }^{*}$ Corresponding author e-mail: niki0611@abv.bg
} 
device is to operate at temperatures from $-40^{\circ} \mathrm{C}$ to $60^{\circ} \mathrm{C}$, it is placed for certain periods of time, and in a certain sequence, at normal temperatures $\left(20^{\circ} \mathrm{C}\right)$ and at end points ambient temperatures and is monitored for changes in its functionality. This approach has proven its effectiveness, but due to the high requirements to the reliability of specialized devices, it is necessary to carry out numerous tests, possibly with a large number of identical sensors, in order to obtain a sample which is sufficient for reliable statistical processing.

The aim of this study is to provide an approach to assess the impact of external influences on the functionality of specialized devices, reducing the number of required tests. For this purpose, models are suggested for describing the probability of failurefree operation of specialized devices in areas close to the limit values of the external influences parameters for which they are designed.

\section{General APPROACH}

Let us assume that a specialized device is designed to operate under extreme influence of a given environmental factor, whose value is denoted by $Z$. Then, in most cases [812], the device is weakly sensitive to the $Z$ variations in relatively large range around its standard values - to values at which the transition to reducing the probability of failure-free operation of the sensor $\left(Z_{s t}\right)$ begins.

In this relatively wide area of external influences variations, the probability of its failure-free operation is relatively stationary and can be denoted by $P_{s t}$. With approaching $Z$ to the limit values for which the device is intended to operate $\left(Z_{e x}\right)$, the probability of its failure-free operation is still high, and in the vicinity of $Z_{e x}$ it starts to tend to its minimum admissible value $P_{e x}$ and then it often sharply decreases (Fig. 2). According the technical requirements and as a result of specialized devices prototype testing, usually the values of $P_{s t}, Z_{s t}, P_{e x}$ and $P_{e x}$ are described in the technical documentation of the device or in the documentation of the producing company [8-10].

Considering the above said, as well as the known dependencies of the reliability characteristics of most real devices [8-11], it could be assumed with sufficient confidence, that in the vicinity of the $Z$ parameters, the effect of its variation on the probability for failure-free operation of the specialized sensor $P(z)$ for the $Z \geq Z_{s t}$ area could be described as an exponential function of the type

$$
P(Z)_{k}=P_{s t} \mathrm{e}^{-a_{1 k}\left(Z-Z_{s t}\right)^{k}},
$$

where $a_{1 k}$ and $k$ are constants.

At $k=1$, equation (1) is transformed into the standard and often used in such cases exponential law, whereby the basis for positioning the exponential curve could be the value of $Z_{s t}$ (Fig. 1). 


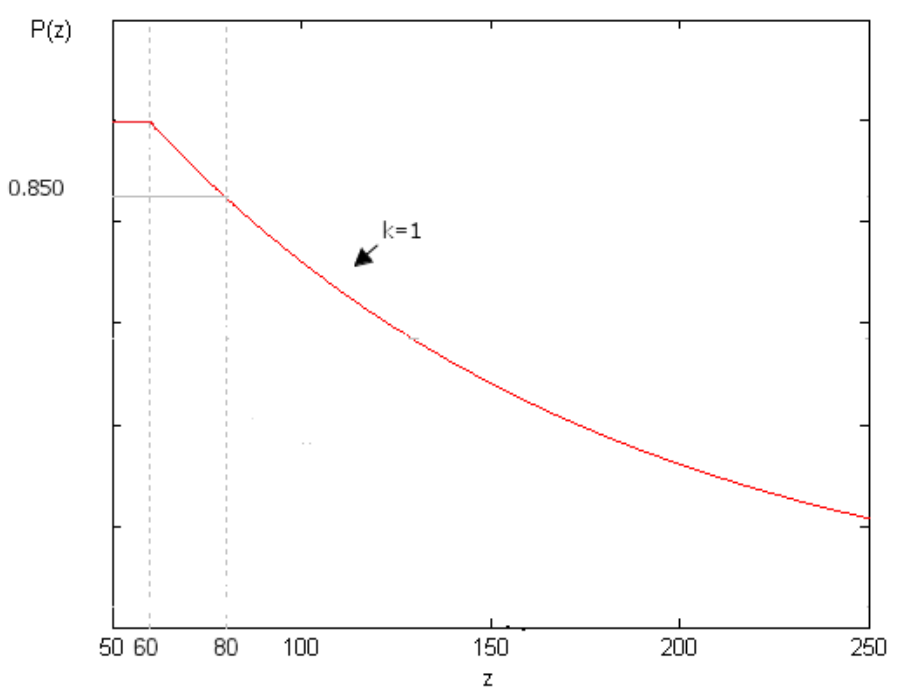

Fig. 1. (Color online) General view of probability of failure-free operation.

In these cases, in general, $P(Z)$ can be assumed to be standard, denoted by $P(Z)_{s t}$ and presented as

$$
P(Z)_{s t}=P_{s t} \mathrm{e}^{-a_{1}\left(Z-Z_{s t}\right)},
$$

where $a_{1}$ is constant.

At $k=2$, equation (1) is transformed into Gauss law, in which case $P(Z)$ can be denoted by $P(Z)_{e x}$. Considering this, the description of the probability of failurefree operation of the sensor can be presented as follows:

$$
P(Z)_{e x}=P_{s t} \exp \left[-a_{2}\left(Z-Z_{s t}\right)^{2}\right],
$$

where $a_{2}$ is constant.

The constants $a_{1}, a_{2}$ and $a_{k}$, and hence the general probability of the failure-free operation of the sensor in the area of $Z \geq Z_{s t}$ for the different $P(z)$ models, can be determined by the following transformations.

At $k=1$, assuming that equation (1) is valid to values of $Z$ equal or close to $Z_{e x}$, we can record that

$$
\begin{aligned}
& P\left(Z_{e x}\right)_{s}=P_{e x}=P_{s t} \exp \left[-a_{1 s}\left(Z_{e x}-Z_{s t}\right)\right], \text { i.e. : } \\
& a_{1}=\frac{\ln \left(P_{s t} / P_{e x}\right)}{\left(Z_{e x}-Z_{s t}\right)} \text { or : }
\end{aligned}
$$




$$
P(Z)_{s}=P_{s t} \exp \left[-\frac{\ln \frac{P_{s t}}{P_{e x}}\left(Z-Z_{s t}\right)}{Z_{e x}-Z_{s t}}\right] .
$$

At Gauss law describing the probability of failure-free operation, assuming that equation (3) is valid to values of $Z$ equal or close to $Z_{e x}$, we can record that:

$$
P\left(Z_{e x}\right)_{g}=P_{e x}=P_{s t} \mathrm{e}^{-a_{2}\left(Z_{e x}-Z_{s t}\right)^{2}},
$$

whence,

$$
a_{2}=\frac{\ln \left(P_{s t} / P_{e x}\right)}{\left(Z_{e x}-Z_{s t}\right)^{2}},
$$

or, replacing $a_{2}$ in equation (2), we obtain

$$
P(Z)_{e x}=P_{s t} \exp \left[-\ln \left(\frac{P_{s t}}{P_{e x}}\right) \frac{\left(Z-Z_{s t}\right)^{2}}{\left(Z_{e x}-Z_{s t}\right)^{2}}\right] .
$$

Similarly, for random values of $k$ we get:

$$
P\left(Z_{e x}\right)_{k}=P_{e x}=P_{s t} \mathrm{e}^{-a_{1 k}\left(Z-Z_{s t}\right)^{k}},
$$

from where we define the constant $a_{k}$ as

$$
a_{k}=\frac{\ln \left(P_{s t} / P_{e x}\right)}{\left(Z_{e x}-Z_{s t}\right)^{k}},
$$

or, replacing $a_{1_{s t}}$ in equation (1), we obtain

$$
P(Z)=P_{s t} \exp \left[-\frac{\ln \left(\frac{P_{s t}}{P_{e x}}\right)\left(Z-Z_{s t}\right)^{k}}{\left(Z_{e x}-Z_{s t}\right)^{k}}\right] .
$$

When $Z$ is replaced with $Z_{s t}$ or with $Z_{e x}$ in equations (6), (9) and (12), for $P(Z)$ values equal to $P_{s t}$ and $P_{e x}$, respectively are obtained.

Apparently, with the increase of $k$ the gradient of $P(Z)$ increases, making the proposed model convenient to use for different in their physical nature specialized sensors.

In the case of unknown values of $P_{s t}$ and $P_{e x}$, finding the value of the constants $a$ and hence - of the general view of $P(Z)_{k}$ - could be determined after conducting experimental studies to find probability values for failure-free operation of the sensor at a value of $Z$ greater than $Z_{s t}$. 
With the proposed $P(Z)$ models of variation (equations (6), (9) and (12)) and a specific value of $k$, it is possible to select a value of external impact parameter for performing tests that is higher than the limit values (by about 25-30\%) [8-12]. This will lead to a reduction in the number of tests necessary to verify the manufacturer's declared probability of failure-free operation of the specialized sensors. The choice of $P(Z)$ and $k$ variation model itself depends on the particular physical characteristic of the external influence $Z$, and in some cases it is possible to use several models simultaneously, with subsequent analysis of the results obtained.

\section{Assessment of the Impact of Extreme External Temperature In- FLUENCES ON THE FUNCTIONALITY OF SPECIALIZED DEVICES}

In this case, it is assumed that there is information about the average probability of the failure-free operation $\left(P_{s t}\right)$ of the specialized device in temperature range with limits $T_{s t}$ and for the limit value of the probability of failure-free operation $\left(P_{e x}\right)$ at limit values of the temperature of the environment in which it is designed to operate $\left(T_{e x}\right)[1,3,6,8,10,12]$.

Then, according to equation (10), in the vicinity of the interval $\left(T_{s t}, T_{e x}+\Delta T\right)$, the dependence of the probability of failure-free operation from the temperature $P(T)$ can be represented as

$$
P(T)_{k}=P_{s t} \mathrm{e}^{-a_{1} k\left(T-T_{s t}\right)^{k}},
$$

where $a_{1_{k}}$ and $k$ are constants.

Using the dependence between $P_{e x}, T_{e x}$ and $P(Z)_{k}$, by transformations (equation (12)) it is possible to determine the value of $a_{1_{k}}$ and by substitution $P(T)$ can be written as

$$
P(T)_{k}=P_{s t} \exp \left[-\frac{\ln \left(\frac{P_{s t}}{P_{e x}}\right)\left(T-T_{s t}\right)^{k}}{\left(T_{e x}-T_{s t}\right)^{k}}\right] .
$$

Most often the impact of temperature on the probability of failure-free operation of the specialized device is modeled by standard exponential law (model M1: at $k=1$ ) or Gauss law (model M2: at $k=2$ we denote $P(T)_{k}$ with $P(T)_{e x}[1,4,5]$.

The type of probability of failure-free operation of the specialized sensor in these two basic models can be written as follows:

M1: Replacing $k$ with 1 and denoting $P(T)_{k}$ with $P(T)_{s t}$ from (2) we obtain

$$
P(T)_{s}=P_{s t} \exp \left[-\frac{\ln \left(\frac{P_{s t}}{P_{e x}}\right)\left(T-T_{s t}\right)}{\left(T_{e x}-T_{s t}\right)}\right] .
$$


M2: Replacing $k$ with 1 and denoting $P(T)_{k}$ with $P(T)_{e x}$ from (2) we obtain

$$
P(T)_{e x}=P_{s t} \exp \left[-\frac{\ln \left(\frac{P_{s t}}{P_{e x}}\right)\left(T-T_{s t}\right)^{2}}{\left(T_{e x}-T_{s t}\right)^{2}}\right] .
$$

In practice, $T_{s t}$ and $T_{e x}$ have two values, usually one in the area of positive and one in the area of negative temperatures. Due to the identical nature of the $P(T)$ variation and in order to ease the description, in the following analyzes we will only consider the area of positive temperatures and, if necessary, will describe the distinctions for the negative temperatures.

Let us analyze the character of the dependencies of the probability of failure-free operation of the specialized device in the two main models of its performance.

Let us assume that the following values are set: $P_{s t}=0.999, T_{s t}=60^{\circ} \mathrm{C}, P_{e x}=$ 0.85 and $T_{e x}=80^{\circ} \mathrm{C}$.

In this case the dependence for $P(T)$ is as follows:

$$
\begin{aligned}
& P(T)_{s t}=0.999 \exp (-0.008076(Z-60)) \text { for } k=1, \\
& P(T)_{e x}=0.999 \exp \left(-0.0004038(Z-60)^{2}\right) \text { for } k=2 .
\end{aligned}
$$

The graphical dependencies of $P(T)_{s t}$ and $P(T)_{e x}$ for this case are shown in Fig. 2. It is seen that at $\Delta T=10^{\circ} \mathrm{C}$ there exists an area of $T$ from $T_{s t}$ to $T_{e x}+\Delta T$, where the two models differ relatively slightly (up to $10 \%$ ).

With a further increase in the impact temperature, the probability of failure-free operation of the specialized device for both models is more and more different and

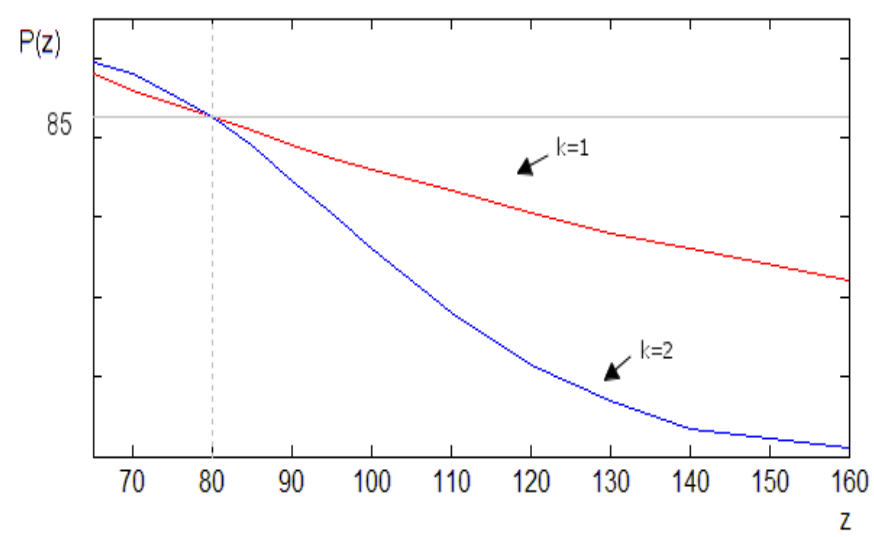

Fig. 2. (Color online) Dependence of $P(T)$ at $k=1$ and $k=2$. 
decreases significantly more slowly in the exponential model (M1) than in the Gauss model (M2). Therefore, for values of $T>T_{e x}+\Delta T$, it is not possible to work by averaging data from both models, but it is necessary to select one of them.

The choice of a particular model could be done by additional tests for other $T$ values greater than $T_{e x}+\Delta T$. As a result of these tests, their corresponding probability of failure-free operation could be determined $[5,6]$.

The choice of model could also be done by expert analysis of the probable influence of temperature on the probability of failure-free operation of the particular type of device. This, however, requires a good knowledge of both the mechanical and hardware composition of the device and the mechanisms of temperature influence on its individual components.

It is known that most specialized devices incorporate electronic and optical components. In these cases the following more significant effects are observed [1,3,8,11]:

- Increasing the temperature results in the real value of the load coefficient being higher than the calculated value, i.e. the electrical mode of operation is changed. Particularly sensitive to high temperatures are active semiconductor elements and microprocessors. In the presence of cooling, especially active, this effect is compensated in a larger range of temperature rise.

- Low temperatures have a detrimental effect on the plastic and rubber parts of devices which crack and lose their insulation properties. At presence of heating, this effect is compensated to a certain extent.

- In cyclic (e.g., round-the-clock) temperature variations in large scale, partial moisture condensation occurs that not only reduces insulation properties, but also increases energy losses in waveguides, reduces sensitivity of receiving devices, and frequency stability of heterodynes, reduces the transmitters power and stability, oxidizes contacts and leads to corrosion of metal parts. When moisture is freezing, cracks in the materials increase, antennas and waveguides are icing and the quality of the switching equipment operation is deteriorating. However, if the device has hydro-absorbent materials, this effect is compensated within certain limits.

The evaluation of these and other factors may lead to the conclusion that, in addition to the use of M1 or M2 model, it is possible, for example, for positive temperatures exceeding $T_{e x}$ the M2 model to be used, and for negative temperatures below $T_{e x}$ - the model M1, or vice versa.

In order to reduce the number of tests in pre-control of specialized devices, it is expedient to choose a model of variation of $P(T)$ and to determine a test value $\left(T_{t}\right)$ in which the probability of their failure-free operation is less of the $P(T)_{e x}$. 
Considering again the temperature range from $T_{e x}$ to $T_{e x}+\Delta T$ it can be assumed that the real model is between 1 and 2. For the example under consideration, this allows the choice of test temperature of the device $T_{t}=90^{\circ} \mathrm{C}$, where the probability of faultless operation is assumed to be approximately 0.74 . In this case, instead of $X_{e x}$ number of tests at temperature $T_{e x}=80^{\circ} \mathrm{C}$, it is possible to carry out $X_{t}$ number of tests at temperature $T_{t}=90^{\circ} \mathrm{C}$. If tests are accepted to be credible until 10 failures are recorded, then because of the fact that $P_{e x}=0.85$, the average number of required $X_{e x}$ tests at temperatures $T_{e x}=80^{\circ} \mathrm{C}$ will be approximately 67. However, if tests are carried out at temperature $T_{t}=90^{\circ} \mathrm{C}$ then because of the fact that the probability of failure-safe operation of the device is approximately equal to 0.75 , the average number of required tests $X_{t}$ till obtaining 10 failures is about 38. It can be seen that the implementation of the models allows for preliminary control of specialized sensors with over $40 \%$ less testing.

\section{Assessment of the Impact of Extreme External Mechanical In- FLUENCES ON THE FUNCTIONALITY OF SPECIALIZED DEVICES}

Mechanical influences destroy or break the construction, fastening parts, plates, solders, connections, adjustments, etc., of the specialized devices.

Most often, mechanical influences are classified as impacts, vibrations, and accelerations $[1,2,4,6,11,12]$.

The impact is a momentary application of external force on the sensor in a very short time interval (milli- or microseconds). The overload that occurs in the impact can be defined as

$$
\tau_{i m}=V / 2 g S,
$$

where $V$ is the velocity of the object at the moment of impact [m/s]; $S$ is the displacement of the object by the impact, or the total magnitude of the residual deformation $[\mathrm{m}] ; g$ is the Earth's acceleration $\left(9.81 \mathrm{~m} / \mathrm{s}^{2}\right)$.

Because $V$ and $S$ are vector quantities, with some simplification it can be claimed that the impacts is characterized by two main components - energy and direction.

Vibrations are prolonged alternating movements caused by forces occurring in reciprocating or rotational motion of the sensor.

With simple harmonic linear vibration, overloading can be represented as

$$
J_{v}=\frac{f_{v}^{2} S}{250},
$$

where $S$ is the amplitude of movement [mm]; $f_{v}$ is the frequency of vibrations [Hz].

The most dangerous are vibrations that resonate at the own frequencies of the devices components (e.g. for radio equipment these are frequencies in the range of 
15 to $500 \mathrm{~Hz}[5,7,8])$. Besides, in most cases, real vibrations are a combination of complex non-linear vibrations.

Because vibrations can be applied to different planes of specialized devices, they can generally be characterized by at least three components - amplitude, frequency and direction of application.

Acceleration is the change in sensor speed, with at least two components - value and direction.

Considering the above, we can assume that a type of mechanical influence is characterized by a number of individual components, each of which has a measurable value $(Z)$.

Generally, the individual components of a particular type of mechanical influence affect complexly the probability of failure-free operation of the sensor, and each of them has its own "weight" and correlation with the influence of the other components. The separate influence and correlation between the influences of the individual components of the mechanical influence are objects of statistical research and are relatively well described in the existing literature. In general, it involves experimental determining the probability of failure-free operation of the device at altering the influence of a single component of the given mechanical effect, while the effects of its other components are fixed $[2,4,6,9,10]$.

As noted, at $k=1$ from equation (1) the dependence of $P(Z)$ is transformed into exponential law, and at $k=2-$ in Gauss law. These two dependencies can be successfully used to model the effects of temperatures, humidity, radiation, etc. in the vicinity of their limit values. In the case of mechanical influences, however, it is advisable to take additional measures to specify the value of $k$ for the particular type of special device. For this purpose it is possible to perform an experimental evaluation of the probability of failure-free operation for $Z=Z_{1}$, where $Z_{1}$ is in the interval $\left(Z_{s t}, Z_{e x}+\Delta Z\right)$, but is higher than $Z_{e x}$. If we denote the value of this probability with $P_{1}$, it follows from equation (4) that:

$$
P_{1}=P\left(Z_{1}\right)=P_{s t} \exp \left[-\ln \left(\frac{P_{s t}}{P_{e x}}\right) \frac{\left(Z_{1}-Z_{s t}\right)^{k}}{\left(Z_{e x}-Z_{s t}\right)^{k}}\right],
$$

i.e.,

$$
\exp \left[\ln \left(\frac{P_{s t}}{P_{e x}}\right) \frac{\left(Z_{1}-Z_{s t}\right)^{k}}{\left(Z_{e x}-Z_{s t}\right)^{k}}\right]=\frac{P_{s t}}{P_{1}}
$$

or

$$
\ln \left(\frac{P_{s t}}{P_{e x}}\right) \frac{\left(Z_{1}-Z_{s t}\right)^{k}}{\left(Z_{e x}-Z_{s t}\right)^{k}}=\ln \left(P_{s t} / P_{1}\right)
$$


whence

$$
\left(\frac{Z_{1}-Z_{s t}}{Z_{e x}-Z_{s t}}\right)^{k}=\frac{\ln \left(\frac{P_{s t}}{P_{1}}\right)}{\ln \left(\frac{P_{s t}}{P_{e x}}\right)} .
$$

By logging the two parts of (24) and making transformations, we get the expression to calculate the value of $k$, which is of the type

$$
\begin{aligned}
& k=\ln \frac{\ln \left(\frac{P_{s t}}{P_{1}}\right)}{\ln \left(\frac{P_{s t}}{P_{e x}}\right)} / \ln \left(\frac{Z_{1}-Z_{s t}}{Z_{e x}-Z_{s t}}\right), \text { or more, } \\
& k=\frac{\ln \left(\ln P_{s t}-\ln P_{1}\right)-\ln \left(\ln P_{s t}-\ln P_{e x}\right)}{\ln \left(Z_{1}-Z_{s t}\right)-\ln \left(Z_{e x}-Z_{s t}\right)} .
\end{aligned}
$$

To demonstrate the above, let us assume that as a characteristic of the probability of failure-free operation of a given specialized device at vibrations of specified frequency are set $P_{s t}=0.999$ at $S_{s t}=60 \mathrm{~mm}, P_{e x}=0.85$ at $S_{e x}=80 \mathrm{~mm}$, and as a result of additional tests it is found that for $S_{1}=90 \mathrm{~mm}$ the probability of faultless operation is $P_{1}=0.75$.

Using equation (25) or (26), for $k$ we get a value approximately equal to 1.415 .

The type of $P(Z)$ function at $k$ equal to 1,2 and 1.415 are shown in Fig. 3.

It can be seen that using models with values of $k=1$ and $k=2$ without further studies and specifying the value of $k$ would result in significant errors, even in areas close to $Z=S_{e x}$.

At the same time, it can be seen from the graph that while for an error of the order of 0.1 for $k=1$ and $k=2$ it is allowed $S$ to exceed $S_{e x}$ by no more than $10 \mathrm{~mm}$,

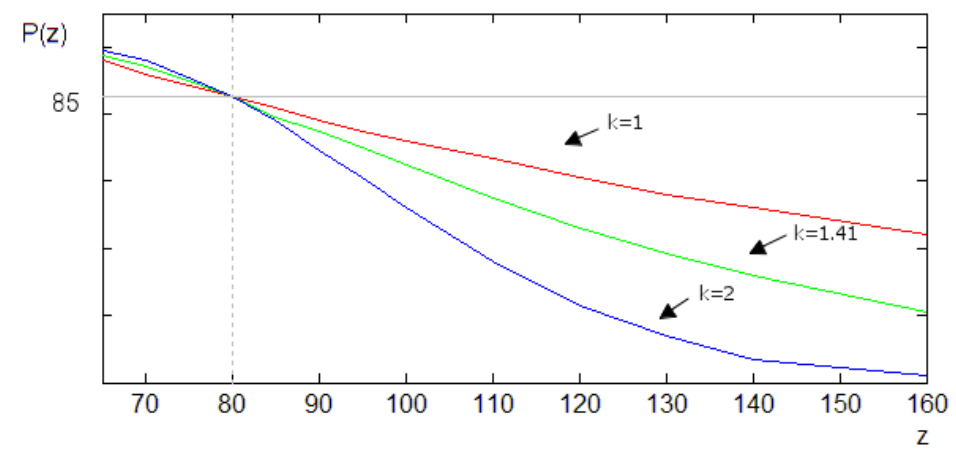

Fig. 3. (Color online) Function $P(Z)$ at $k=1, k=1.415$ and $k=2$. 
at $k=1.415$ such error (compared to the other two models) is achieved when $S$ exceeds $S_{e x}$ by about $20 \mathrm{~mm}$.

At a specified value of $k$, it is possible the dependence of the probability of failurefree operation of the sensor from $S$ at vibration with specified frequency to be simplified and for the examined example $(k=1.415)$ to obtain the type

$$
\begin{aligned}
& P(Z)=0.999 \exp \left(-\frac{0.1615(Z-60)^{k}}{(20)^{k}}\right), \text { or } \\
& P(Z)=0.999 \mathrm{e}^{\left(-0.00233(Z-60)^{1.415}\right)} .
\end{aligned}
$$

It can be affirmed that at specified frequency of vibrations equation (12) approximates relatively well the dependency of the probability of failure-free operation of the examined sensor from $S$ within the range of $60 \mathrm{~mm}$ to $90-100 \mathrm{~mm}$. Therefore, when performing tests, according to the capabilities of the laboratory equipment, it is possible to use random vibration amplitudes within the specified range, and then to calculate the value of $P(Z)$. It is also possible to perform tests at the maximum values of $S(90-100 \mathrm{~mm}$ ), thereby significantly reducing (over $40 \%$ ) the number of tests to preliminary verify the reliability of the declared probability of the sensor failure-free operation.

\section{CONCLUSIONS}

An approach is proposed to accelerate the tests intended to assess the impact of extreme external influences on the functionality of specialized devices. The approach is applicable when there is data about the stationary and the limit value of the probability of failure-free operation of the specialized devices, as well as their respective values under a specified external impact parameter. The approach is further developed and demonstrated for the most common extreme external influences - both temperature and mechanical - in which the specialized devices operate. It has been found that the proposed approach could lead to a $40 \%$ reduction in the number of tests required, which in turn is associated with significant savings of time and financial resources. Although the limitations introduced refer to a wide range of practical situations, before using the proposed approach it is necessary to assess whether the hypotheses of its applicability are adequate to the particular device and the environment of use.

\section{REFERENCES}

[1] A.K. Tyagi, S. BanerJee (Ed.) (2017) "Materials under Extreme Conditions: Recent Trends and Future Prospects". Elsevier, eBook ISBN: 9780128014424.

[2] D.D. ReYnolds (2016) "Engineering Principles of Mechanical Vibration", 4th ed. Bloomington, Indiana, USA: Trafford On Demand Publishing, ISBN 978-1490714370. 
[3] C.M. Branco, R.O. Ritchie, V. SkleničKa (1996) "Mechanical behavior of materials at high temperature". Springer. ISBN 978-0-7923-4113-0.

[4] A. CRAWFORD (2010) "Simplified Handbook of Vibration Analysis". Wellington, FL, USA: Computational Systems, ISBN-10:9994616935.

[5] J. Fraden (2010) "Handbook of Modern Sensors: Physics, Designs, and Applications". Springer.

[6] MIL-STD-810G: (31.10.2008) "Environmental Engineering Considerations and Laboratory Tests". Department of Defense Test Method Standard. USA.

[7] "Perimeter Security Sensor Technologies Handbook". (1997) Defense Advanced Research Projects Agency (DARPA), USA.

[8] V.A. Petrov (1993) "Physical Basis for Predicting the Durability of Structural Materials". Moscow: Politechnika (in Russian).

[9] V.G. Vorobyov, V.V. Glukhov, Yu.V. Kozlov, V.D. Konstantinov, I.M. SINDEEV (1984) "Diagnostics and Forecasting of the Technical Condition of Aviation Equipment. Manual for universities of civil aviation”. Moscow: Transport (in Russian).

[10] V.V. Nosov (2012) "Diagnostics of Machines and Equipment". St. Petersburg: LanTrade, ISBN 9785811412693 (in Russian).

[11] T. BAlBuzAnov (2012) Methods for Evaluating the Reliability of a Technical Object. Research Works of the University of Russe 51(4) 120-124. (in Bulgarian)

[12] V.V. Nalimov, N.A. Chernova (1965) "Statistical Methods for Planning Experimental Experiments”. Moscow: Nauka (in Russian). 